\title{
A Focused Ion Beam Specimen Preparation Method to Minimize Gallium Ion Concentration in Copper Atom-Probe Tomography Specimen Tips
}

\author{
R. Prakash Kolli ${ }^{1,2}$ and Frederick Meisenkothen ${ }^{2}$ \\ ${ }^{1}$ Department of Materials Science and Engineering, University of Maryland, College Park, MD, USA \\ ${ }^{2}$ Materials Measurement Science Division, National Institute of Standards and Technology, \\ Gaithersburg, MD, USA
}

Atom-probe tomography (APT) is a quantitative technique that permits three-dimensional (3-D) spectroscopic characterization of interfaces and other nanometer-scale features within a material. Specimens for atom-probe tomography (APT) analysis of semiconductor devices and nanostructured materials are typically fabricated employing a focused ion beam (FIB) instrument [1-3] or a dual-beam FIB instrument [4-6]. One method used to fabricate APT specimen tips from bulk material is an in situ lift-out method that is adapted from a standard lift-out method for transmission electron microscopy (TEM) specimens [7]. This method employs a series of sequential steps to create a specimen tip with a radius $<\sim 100 \mathrm{~nm}$ that is appropriate for APT analysis. The employment of a FIB instrument provides several advantages over conventional two-step electro-polishing methods to include applicability to all materials, reduction of preferential etching in multi-phase materials, reduction in use of hazardous chemicals, and increased control during specimen preparation. It also permits fabrication of APT specimen tips from alternative material geometries, such as thin sheets, flakes, ribbons, powders, and thin films that may be difficult to work with when using electro-polishing methods $[5,6]$. However, one well-known disadvantage of using the FIB to prepare specimen tips is the implantation of gallium ${ }^{+}\left(\mathrm{Ga}^{+}\right)$ ions into the tip material. Ga ion implantation is observed as artifact peaks in the mass spectra at a massto-charge-state $(\mathrm{m} / \mathrm{n})$ ratio of 69 and 71 . Excessive $\mathrm{Ga}^{+}$ion implantation may hinder the compositional analysis of semiconductor devices and nanostructured materials. Additionally, it is known that copper $(\mathrm{Cu})$, which is a commercially important material for semiconductor devices and other nanostructured materials, exhibits an irregular surface topography under a wide range of milling conditions and increased redeposition of sputtered material [7]. Thus, a FIB-based technique that minimizes $\mathrm{Ga}^{+}$ion implantation and creates consistent specimen geometries for $\mathrm{Cu}$ is advantageous for APT analysis.

An in situ lift-out method to fabricate Cu APT specimen tips employing a dual-beam FIB instrument is demonstrated. The specimen preparation method permits fabrication of approximately reproducible specimen tip geometries with low implanted $\mathrm{Ga}^{+}$ion concentrations. It also allows the extraction of specimens from site-specific locations or non-site-specific locations, and also from the surface, shallow sub-surface, or deeper locations. The method defines and extracts a region of interest (ROI) by (1) depositing a platinum (Pt) protective layer; (2) trench milling around the Pt protective layer to create a rectangular wedge, (3) extracting the wedge using an in situ micromanipulator needle, (4) mounting sections of the wedge on standardized Si posts of a microtip array (obtained from Cameca [8]), and (5) then introduces a five-step annular milling sequence. The prescribed sequence of beam currents and radii when combined with a $1 \mu \mathrm{m}$ thick Pt protective cap, consistently minimizes $\mathrm{Ga}^{+}$ion implantation to $<\sim 0.15$ at.\% as measured by APT in the specimen tips. This Ga concentration is less than the quantities reported in the existing literature for other in situ lift-out methods used to fabricate APT specimen tips. The method also creates approximately reproducible specimen tip geometries with a tip radius $<\sim 50 \mathrm{~nm}$. 


\section{References:}

[1] D. J. Larson et al., Ultramicrosc. 79, (1999) 287.

[2] D. J. Larson et al., Microsc. Microanal. 7, (2001) 24.

[3] G. B. Thompson et al.,Ultramicrosc. 100, (2004) 25.

[4] K. Thompson et al., Ultramicrosc. 107, (2007) 131.

[5] M. K. Miller et al., Ultramicrosc. 102, (2005) 287.

[6] M. K. Miller and K. F. Russell, Ultramicrosc. 107, 761 (2007).

[7] L. A. Giannuzzi et al., in Introduction to Focused Ion Beams, edited by L. A. Giannuzzi and F. A. Stevie (Springer US, 2005), pp. 201-228.

[8] Certain commercial equipment, instruments, or materials are identified in this paper in order to specify the experimental procedure adequately. Such identification is not intended to imply recommendation or endorsement by the National Institute of Standards and Technology, nor is it intended to imply that the materials or equipment identified are necessarily the best available for the purpose.

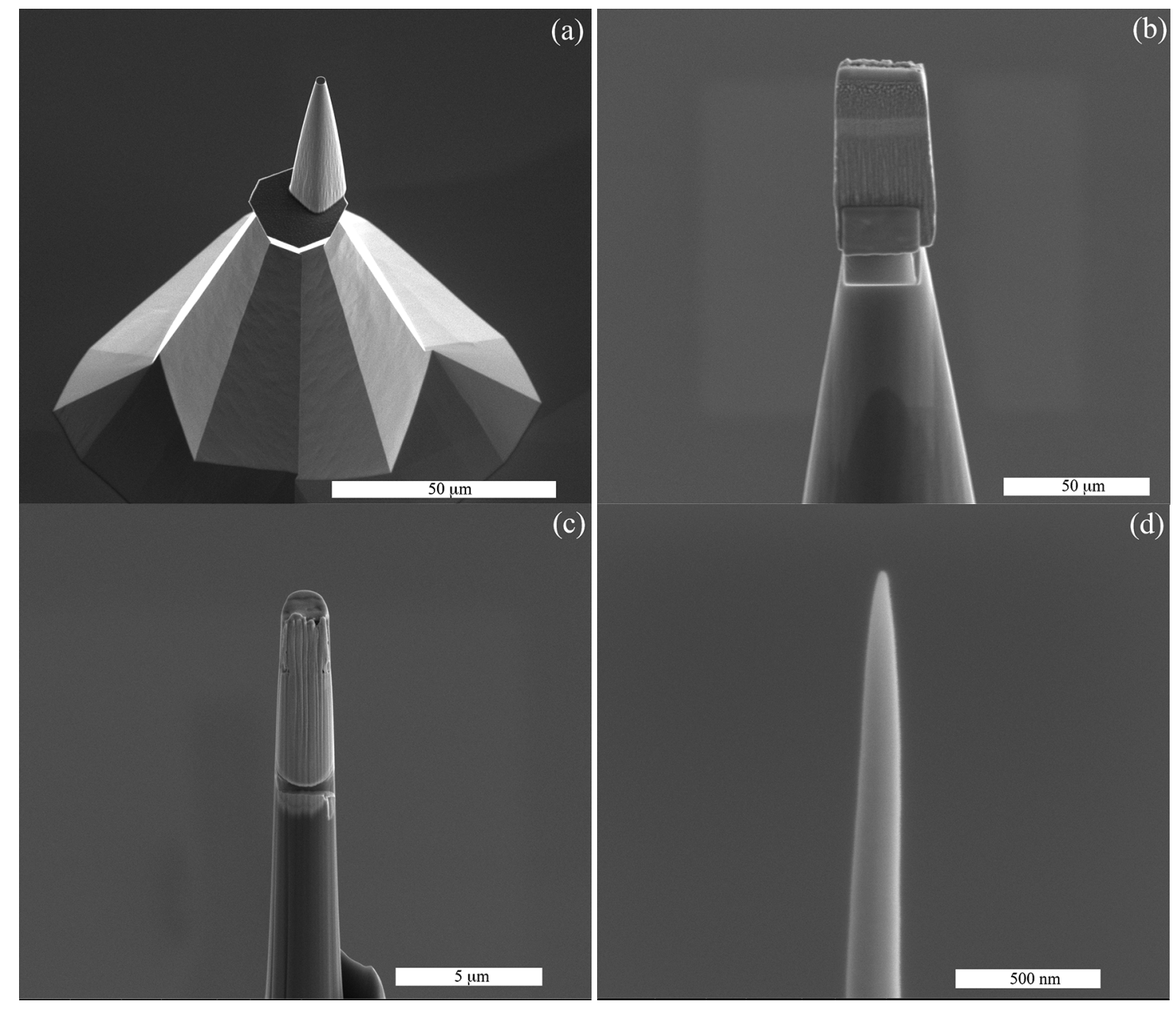

Figure 1. An illustration of the partial sequence of steps to fabricate an APT specimen tip with low implanted $\mathrm{Ga}^{+}$ion concentration including (a) the silicon (Si) post from a micro-tip array, (b) an APT specimen tip blank that was positioned onto the flat surface of the post by an in situ micromanipulator needle and attached with Pt deposition, (c) the initial annular ion beam milling step, and (d) the sharpened APT specimen tip that has a radius $<\sim 50 \mathrm{~nm}$. 